\title{
The merging/AGN connection
}

\section{Ionization of the circumnuclear regions}

\author{
S. F. Sánchez ${ }^{1, \star, \star \star}$, T. Becker ${ }^{1}$, B. Garcia-Lorenzo ${ }^{2}$, C. R. Benn ${ }^{3}$, L. Christensen ${ }^{1}$, A. Kelz $^{1}$, \\ K. Jahnke ${ }^{1}$, and M. M. Roth ${ }^{1}$ \\ 1 Astrophysikalisches Institut Potsdam, And der Sternwarte 16, 14482 Potsdam, Germany \\ e-mail: ssanchez@aip.de \\ 2 Instituto de Astrofísica de Canarias, 38205 La Laguna, Tenerife, Spain \\ 3 Isaac Newton Group, Apt. 321, 38700 Santa Cruz de La Palma, Spain
}

Received 17 September 2004 / Accepted 17 November 2004

\begin{abstract}
We report the first results of a study of a sample of 20 galaxy mergers/interacting systems, using the VIMOS and PMAS integral field spectrographs. For each object, we extracted the integrated spectrum of the central regions and analyzed the ionization state using classical diagnostic diagrams (Veilleux \& Osterbrock 1987). There is evidence of AGN ionization in 4 of the objects, i.e. $20 \%$ of the sample, a considerably higher fraction than found in previous studies ( 4\%). An increase of the nuclear activity with the far infrared temperature may account for the difference.
\end{abstract}

Key words. galaxies: active - galaxies: interactions - galaxies: irregular - galaxies: ISM

\section{Introduction}

Interactions and mergers can strongly affect galaxy evolution. Since the early studies by Toomre \& Toomre (1972) and Larson \& Tinsley (1978), it is generally believed that interactions can trigger starburst processes in galaxies, via tidal effects. The fact that interacting galaxies are more luminous in the far infrared (FIR) (e.g. Sanders \& Mirabel 1996) and the radio (e.g. Hummel 1981), and that they have larger $\mathrm{H} \alpha$ equivalent widths (e.g. Kennicutt et al. 1987) supports this hypothesis. The star formation is found to be stronger in the nucleus, although it is also enhanced in the disk. A widely accepted idea, supported by simulations, is that galaxy interactions induce flows of gas from the outer parts of the galaxy and/or the companion into the inner regions through loss of the angular momentum induced by tidal forces (e.g. Mihos \& Hernquist 1996).

It has long been suggested that strong interactions and mergers may trigger nuclear activity. Many authors have found that quasar host galaxies show distorted morphologies, reminiscent of past merging events (e.g. McLeod \& Rieke 1994a,b; Bahcall et al. 1997; Sánchez \& González-Serrano 2003; Jahnke et al. 2004). However, other authors found no evidence of past

^ Based on observations made with ESO Telescopes at the Paranal Observatory under programme ID 71.B-3055(A).

$\star \star$ Visiting Astronomer, German-Spanish Astronomical Centre of Calar Alto. interactions (e.g. Dunlop et al. 2003). It is not clear if the fraction of AGN host galaxies undergoing interactions is really larger than the fraction of inactive galaxies undergoing interactions, or how the timescales of the morphological relaxation and the AGN ignition relate (e.g. Sánchez et al. 2004). However, it is clear that some AGN, if not all, are generated by mergers/interactions between galaxies (Canalizo \& Stockton 2001; Cattaneo \& et al. 2004).

The fraction of AGNs in merging systems increases with the FIR luminosity, being $\sim 30 \%$ for the extreme case of the ultraluminous infrared galaxies (ULIRGs) (Bushouse et al. 2002), with FIR luminosity $L_{\mathrm{FIR}}>10^{12} L_{\odot}$. Sanders et al. (1988) suggested an evolutionary scenario, in which major mergers may allow the infall of gas towards the nuclear regions, triggering nuclear star formation and igniting an AGN. Both the AGN and the star formation heat the dust, and the object is observed as a ULIRG. Colina et al. (2001) proposed a modification of this scenario in which cool ULIRGs would be generated during the merging of two or more low-mass sub$L^{*}$ spirals, and warm ULIRGs in the merging of an $L^{*}$ galaxy with a substantially less massive galaxy or during the merging of two intermediate-mass spirals. Under this hypothesis only warm ULIRGs would evolve/host an AGN, while cool ULIRGs would be heated by star formation.

At lower luminosities, $L_{\mathrm{FIR}} \sim 10^{10-11} L_{\odot}$, there is no evidence of an excess of AGNs among interacting/merging 
systems compared to isolated galaxies (e.g. Dahari 1985; Keel et al. 1985; Bushouse et al. 1988; Sekiguchi \& Wolstencroft 1992; Liu \& Kennicutt 1995; Pastoriza et al. 1999; Donzelli \& Pastoriza 2000; Bergvall et al. 2003). This deficiency could be partially explained by the presence of strong circumnuclear star formation that can blur the AGN signatures (Pastoriza et al. 1999; Donzelli \& Pastoriza 2000). There is significantly more nuclear star formation in interacting galaxies, compared to isolated ones (e.g. Bergvall et al. 2003). This nuclear star formation increases with the FIR temperature (Bushouse et al. 1988; Bergvall et al. 2003). If the scenario proposed by Colina et al. (2001) is valid for lower-luminosity objects, the nuclear SFR and the probability of generating an AGN could be larger in warm FIR sources than in cool ones. de Grijp et al. (1985) and van den Broek et al. (1991) already noticed an increase of the number of AGNs with the FIR temperature, using slit spectroscopy.

Most previous studies of this topic were based on samples of interacting/merging galaxies selected by morphology (e.g. Donzelli \& Pastoriza 1997; Bergvall et al. 2003), without considering the FIR properties. As a consequence, these samples contain mainly cool FIR sources $\left(\log _{10}\left(f_{60 \mu} / f_{100 \mu}\right) \sim\right.$ $-0.4)$. We selected a sample of 20 IRAS sources with evidence of interactions/major merging (Sánchez et al. 2004b, Paper I), with similar FIR luminosities to previous samples, $L_{\mathrm{FIR}} \sim 10^{10-11} L_{\odot}$, but warmer $\left(\log _{10}\left(f_{60 \mu} / f_{100 \mu}\right) \sim-0.25\right)$. Since the use of slit spectroscopy can bias the results (Paper I), we obtained integral-field spectra of all them to explore the connection between galaxy interactions and nuclear activity.

\section{Observations and data reduction}

The spectra were taken with the integral-field units (IFUs) of VIMOS (LeFevre et al. 2003), 17 objects, and PMAS (Roth et al. 2000), 3 objects. The VIMOS observations were made on the nights of the 9th, 10th, 22th, 23th, 24th and 26th of April 2003 at the VLT/UT3, in clear sky conditions, with an average seeing $\sim 1.6^{\prime \prime}$. We obtained, for each object, 3 exposures of $300 \mathrm{~s}$ using each of the low-resolution red ( $\mathrm{LRr}$ ) and blue $(\mathrm{LRb})$ grisms. With this setup we cover the wavelength ranges

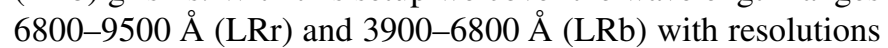
of $7.14 \AA /$ pixel (LRr) and $5.35 \AA /$ pixel (LRb), respectively. The VIMOS IFU contains a focal-plane array of 6400 lenslets each of size $0.67^{\prime \prime} \times 0.67^{\prime \prime}$ (in the low-resolution mode) distributed on a regular grid. The final field-of-view was $54^{\prime \prime} \times 54^{\prime \prime}$, which allows us to cover the different components within the IRAS aperture $\left(\sim 1^{\prime}\right)$. In two cases (IRAS 10219-2828 and IRAS 12110-3412) we obtained data for only one grism ( $\mathrm{LRr}$ and $\mathrm{LRb}$, respectively).

The PMAS data were taken during the nights of the 28th of April, and 5th and 8th of August 2003 at the 3.5-m telescope of the Calar Alto Observatory. The observations were made in poor weather conditions as a back-up project for these nights. We used the V300 grating which covers the wavelength range $\sim 4000-7700 \AA$ with a resolution of $3.3 \AA /$ pix. The exact wavelength range depends on the rotation angle selected for the grating, which changes from object to object. The PMAS focal plane contains an array of 256 lenslets each of size $0.5^{\prime \prime} \times 0.5^{\prime \prime}$

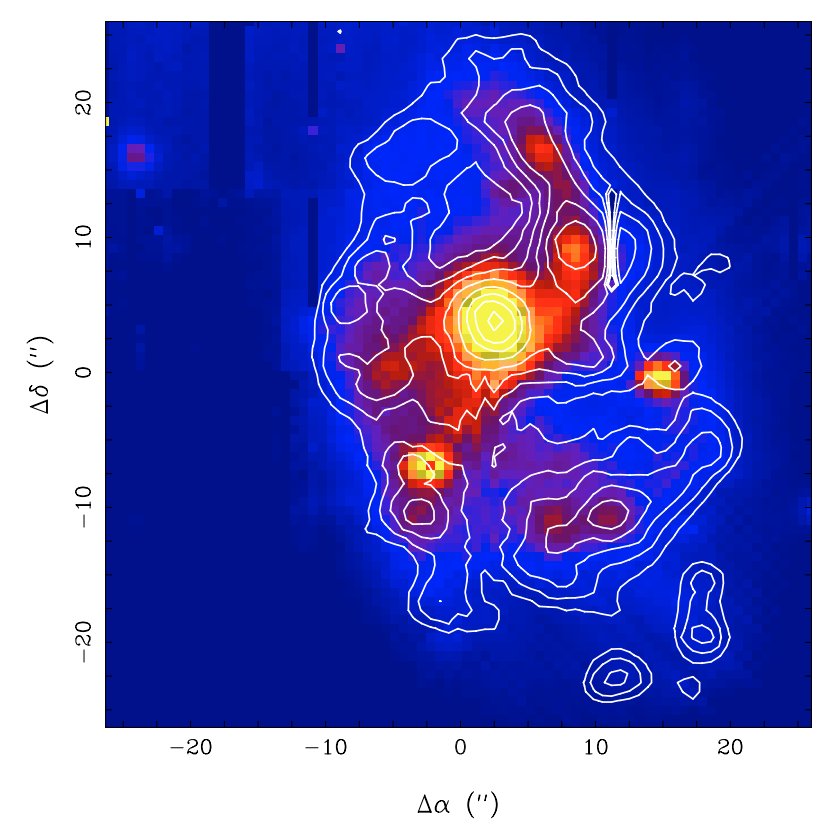

Fig. 1. Intensity map of the continnum emission of IRAS 13477-4848 at wavelengths $\sim 6477-6591 \AA$, extracted from the VIMOS datacube. The contours show the emission in $\mathrm{H} \alpha+[\mathrm{NII}]$ on a logarithm scale, obtained by subtracting the continuum emission from that at wavelengths centred on the emission lines ( 6612-6670 ̊).

(in the standard configuration) distributed on a square grid. The field-of-view is $8^{\prime \prime} \times 8^{\prime \prime}$, i.e. too small to cover the IRAS aperture with a single pointing. Different mosaic patterns were used for each object to cover the central regions and different regions of interest (e.g., tidal tails). Due to the variable atmospheric conditions different exposure times were used for each object and each night, to obtain similar signal-to-noise levels in all the frames. The exposure times ranged from $1200 \mathrm{~s}$ per pointing for IRAS $16365+4202(05 / 08 / 03)$ to 5400 s for IRAS $12110+1624$ (28/04/03).

Both the VIMOS and PMAS data were reduced using the $\mathrm{P} 3 \mathrm{~d}$ reduction package, written in IDL (Becker 2002). Although initially written to reduce PMAS data, it was slightly modified to reduce data from other IFUs, such as VIMOS (e.g. Sánchez et al. 2004a). The expected locations of the spectra were traced on a continuum-lamp exposure obtained before each target exposure. After bias subtraction, the target spectra were extracted by adding the signal from the 5 pixels around the central traced pixel. The continuum-lamp exposure was also used to determine the fiber-to-fiber response of the instrument at each particular wavelength (the so-called flat-spectra). These flat-spectra were used to homogenize the response of all the fibers. Wavelength calibration was carried out via observation of emission-line lamps, also obtained before the science exposures. The accuracy of the wavelength calibration was checked using sky emission lines, and has standard deviation $1.2 \AA$ (VIMOS-LRr), $1.0 \AA$ (VIMOS-LRb) and $0.5 \AA$ (PMAS). Different exposures taken at the same pointing were combined using IRAF tasks. A $3 \sigma$ clipping algorithm was used to remove possible cosmic rays. The different PMAS pointings were mosaiced into a single frame, using the Euro3D data format, as described in Sánchez et al. (2004a). 
Further analysis was carried out using E3D (Sánchez 2004), and our own fitting routines (Sánchez et al., in preparation). An average sky spectrum, taken from areas uncontaminated by target spectra, was subtracted from the target spectra.

\section{Analysis and results}

\subsection{Extraction of the nuclear spectra}

Figure 1 shows an example of the reduced data: a continuum-intensity map of IRAS $13477-4848$ at wavelengths 6477-6591 A, extracted from the VIMOS datacube. For each object we extracted the spectrum of the central region, by coadding the spectra within a box aperture of size $2^{\prime \prime} \times 2^{\prime \prime}$ (approximately the central kiloparsec, at the redshift of these objects). Whenever the intensity peak of the continuum emission was not coincident with that of the emission line, we centered our aperture on the latter to increase the signal-to-noise ratio. In most cases both peaks were coincident within a couple of arcsec. This is illustrated in Fig. 1, where the $\mathrm{H} \alpha+[\mathrm{NII}]$ intensity contours are superimposed on a continuum image.

Six of the 20 objects have two well-separated components. with two clear intensity peaks. The spectra of four of them show emission lines in both components. Emission lines are absent in the remaining two. The extracted spectra of the nuclear regions of the two components have similar characteristics. Hereafter we restrict our discussion to the properties of the spectrum of the brighter component.

\subsection{Ionization mechanism}

The final dataset consists of the spectra of the nuclear regions of 20 interacting/merging galaxies. Of these, 18 cover a wavelength range which includes some of the most important emission lines for ionization diagnostics: i.e., $\mathrm{H} \beta$, [OIII] $\lambda 4959$, [OIII] $\lambda 5007, \mathrm{H} \alpha,[\mathrm{NII}] \lambda 6584,[\mathrm{NII}] \lambda 6548,[\mathrm{SII}] \lambda 6717$ and [SII] 26731 . A variety of other emission lines were detected in many of these spectra. A detailed description of the spectrum of each object will be presented elsewhere (Sanchez et al., in preparation). We fitted the line profiles to derive the integrated flux of each emission line, using our own software. A single gaussian was fitted to each line, using a polynomial function to characterize the continuum. The $\mathrm{H} \alpha$-[NII] system and the $[\mathrm{SII}]$ doublet were blended in the LRr VIMOS data. For proper deblending, the minimun line-width was fixed to be the width measured in isolated sky emission lines (i.e., the instrumental width). Our software allows definition of emission line systems, i.e., a kinematically coupled set of emission lines with the same width and heliocentric velocity. This was essential for accurate deblending of the lines. After deriving the flux of each line, the $[\mathrm{OIII}] \lambda 5007 / \mathrm{H} \beta,[\mathrm{NII}] \lambda 6583 / \mathrm{H} \alpha$ and [SII] $\lambda 6717+6731 / \mathrm{H} \alpha$ line ratios were calculated.

Figure 2 shows the basic diagnostic diagram, [OIII] $\lambda 5007 / \mathrm{H} \beta$ vs. [NII] $\lambda 6583 / \mathrm{H} \alpha$ (Veilleux \& Osterbrock 1987), for the 18 objects observed with spectral information in both wavelength regions (solid squares). The open squares near the edge of the plot represent two objects (IRAS 10219-2828 and IRAS 12110-3412) with data only for one VIMOS grism

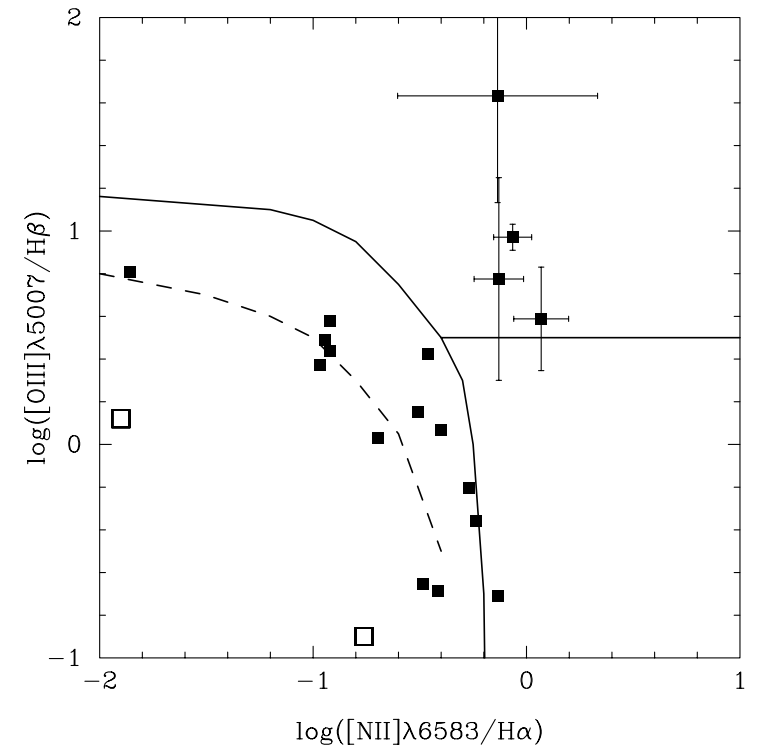

Fig. 2. $[\mathrm{OIII}] \lambda 5007 / \mathrm{H} \beta$ line ratio as a function of $[\mathrm{NII}] \lambda 6583 / \mathrm{H} \alpha$ for the 18 objects with both ratios measured solid squares). The open squares indicate the two objects with data only for one VIMOS grism (LR-red or LR-blue). They have been plotted with an arbitrary value of the un-measured line ratio (i.e. at the edge of the figure). The solid lines show the separation between ionization due to star formation (bottom-left), AGN (top-right) and LINERs (bottom-right). The approximate locus of star-forming objects reported by Veilleux \& Osterbrock (1987), is indicated with a dashed line.

(LR-red or LR-blue). The solid lines show the classical separation between ionization due to star formation (bottom-left), AGNs (top-right) and LINERs (bottom-right). The boundary curve between AGNs and star forming regions is taken from Veilleux \& Osterbrock (1987). A separation between AGNs and LINERs at a ratio of [OIII] $/ \mathrm{H} \beta=3$ was assumed, following earlier results in the literature (e.g. Donzelli \& Pastoriza 2000; García-Lorenzo et al. 2001).

The majority of the objects in our sample (14 out of 20) have nuclear emission features characteristic of star forming regions. However, for a significant fraction of the objects (4 out of $20,20 \%$ ), the nuclear emission is dominated by an AGN: IRAS 16330-6820, IRAS 12193-3942, IRAS 13031-5717 and IRAS $16365+4202$. We include the $1 \sigma$ error bars for these objects in Fig. 2. As a check, we repeated the analysis using the $[\mathrm{OIII}] \lambda 5007 / \mathrm{H} \beta$ vs. [SII] $\lambda 6717+6731 / \mathrm{H} \alpha$ diagnostic diagram. We find the same distribution, reinforcing the result from Fig. 2. As expected from ionization models (e.g. Osterbrock 1989), there is a correlation between the [NII] $\lambda 6583 / \mathrm{H} \alpha$ and the [SII] $\lambda 6717+6731 / \mathrm{H} \alpha$ line ratios in our sample, with a dispersion dominated by measurement errors. The errors in the $[\mathrm{NII}] \lambda 6583 / \mathrm{H} \alpha$ ratio are dominated by residual errors in the line-deblending procedure, while those of [SII] $\lambda 6717+6731 / \mathrm{H} \alpha$ (less affected by deblending) are dominated by the lower signal-to-noise ratio of [SII] $\lambda 6717+6731$.

For the two objects with only one diagnostic line ratio measured, the nature of the ionization is uncertain, although one of them, IRAS 12110-3412, is clearly not an AGN. 


\section{Discussion and conclusions}

As noted above, most studies of the ionization mechanisms in the nuclear regions of merging/interacting galaxies are based on studies of cool FIR sources. These studies found a small fraction of objects with AGN-like ionization, typically $\sim 4 \%$ (e.g. Pastoriza et al. 1999; Bergvall et al. 2003). By contrast, we find a significantly higher fraction of AGNs $(\sim 20 \%)$, in a sample of warmer FIR merging/interacting galaxies. This may indicate that the scenario proposed by Colina et al. (2001) (that only warm ones host an AGN), is valid also at lower IR luminosities $L_{\mathrm{FIR}}$.

Our current sample corresponds to only $\sim 6 \%$ of all interacting systems with measured redshift in the IRAS point-source sample (Sánchez et al. 2004b). How general is our result? To address this question we compared our sample with that of Bergvall et al. (2003). As already mentioned, the two samples cover the same range of FIR luminosities (indistinguishable, according to a Kolmogorov-Smirnov test). However, the ranges of FIR temperatures are different, with our sample being warmer (the null hyphothesis, that both samples were drawn from the same parent population, can be rejected at the $0.13 \%$ level). If we add the objects from Bergvall et al. (2003) within the IRAS point source catalogue (Joint IRAS Science 1994) to our sample, we end up with 77 objects. Splitting this sample into cool $\left(f_{60 \mu} / f_{100 \mu}<-0.3\right)$ and warm $\left(f_{60 \mu} / f_{100 \mu}>-0.3\right)$ FIR sources, we found that only $4 \%$ ( 2 out of 50 ) of the cool objects harbour an AGN, in compared with 15\% (4 out of 27) of the warm ones. That is, we still find that the fraction of AGN increases with the FIR temperature. We found similar results when comparing with other samples (e.g. Donzelli \& Pastoriza 1997, 2000).

The probability of igniting an AGN seems to be higher in warm FIR sources, over a large range of FIR luminosities. According to Colina et al. (2001), these objects are the result of a merger either between a large galaxy and a small galaxy or between two galaxies of similar size. A possible explanation of this result could be that apart from the fuel supply provided by the interaction/merging, the presence of a massive blackhole is required to ignite an AGN. Massive black-holes are found in massive galaxies, due to the black-hole/bulge-mass relation (e.g., Magorrian et al. 1998; Kormendy \& Gebhardt 2001). Therefore, only galaxy mergers with one progenitor harbouring a massive black hole (i.e. a massive galaxy) may (re-)ignite an AGN, and those are the mergers that generate a warm FIR source.

Acknowledgements. We thank Dr. E. Laurikainen and Dr. N. Bergvall for providing us with their dataset. We thank Dr. A. Stockton, who refereed this article, and help us to improve it. This project is founded by the Euro3D RTN on IFS, funded by the EC under contract No. HPRN-CT-2002-00305. L. Christensen acknowledges support by the German Verbundforshung associated with the ULTROS project, grant No. 05AE2BAA/4.

\section{References}

Bahcall, J. N., Kirhakos, S., Saxe, D. H., \& Schneider, D. P. 1997, ApJ, 479, 642

Becker, T. 2002, Ph.D. Thesis, University of Potsdam, Germany

Bergvall, N., Laurikainen, E., \& Aalto, S. 2003, A\&A, 405, 31

Bushouse, H. A., Borne, K. D., Colina, L., et al. 2002, ApJS, 138, 1

Bushouse, H. A., Werner, M. W., \& Lamb, S. A. 1988, ApJ, 335, 74

Canalizo, G., \& Stockton, A. 2001, ApJ, 555, 719

Cattaneo, A. , Combes, F. , Colombi, S., et al. 2004, MNRAS, submitted

Colina, L., Borne, K., Bushouse, H., et al. 2001, ApJ, 563, 546

Dahari, O. 1985, ApJS, 57, 643

de Grijp, M. H. K., Miley, G. K., Lub, J., \& de Jong, T. 1985, Nature, 314,240

Donzelli, C. J., \& Pastoriza, M. G. 1997, ApJS, 111, 181

Donzelli, C. J., \& Pastoriza, M. G. 2000, AJ, 120, 189

Dunlop, J. S., McLure, R. J., Kukula, M. J., et al. 2003, MNRAS, 340, 1095

García-Lorenzo, B., Arribas, S., \& Mediavilla, E. 2001, A\&A, 378, 787

Hummel, E. 1981, A\&A, 96, 111

Jahnke, K., Kuhlbrodt, B., \& Wisotzki, L. 2004, MNRAS, 352, 399

Joint IRAS Science, W. G. 1994, VizieR Online Data Catalog, 2125, 0

Keel, W. C., Kennicutt, R. C., Hummel, E., \& van der Hulst, J. M. 1985, AJ, 90, 708

Kennicutt, R. C., Roettiger, K. A., Keel, W. C., van der Hulst, J. M., \& Hummel, E. 1987, AJ, 93, 1011

Kormendy, J., \& Gebhardt, K. 2001, in AIP Conf. Proc., 20th Texas Symp. relativistic Astrophys., 586, 363

Larson, R. B., \& Tinsley, B. M. 1978, ApJ, 219, 46

LeFevre, O., Saisse, M., Mancini, D., et al. 2003, Proc. SPIE, 4841, 1670

Liu, C. T., \& Kennicutt, R. C. 1995, ApJS, 100, 325

Magorrian, J., Tremaine, S., Richstone, D., et al. 1998, AJ, 115, 2285

McLeod, K. K., \& Rieke, G. H. 1994a, ApJ, 420, 58

McLeod, K. K., \& Rieke, G. H. 1994b, ApJ, 431, 137

Mihos, J. C., \& Hernquist, L. 1996, ApJ, 464, 641

Osterbrock, D. E. 1989, Astrophysics of gaseous nebulae and active galactic nuclei (University Science Books)

Pastoriza, M. G., Donzelli, C. J., \& Bonatto, C. 1999, A\&A, 347, 55

Roth, M. M., Bauer, S., Dionies, F., et al. 2000, in Proc. SPIE, 4008, 277

Sánchez, S. F. 2004, AN, 325, 167

Sánchez, S. F., Becker, T., \& Kelz, A. 2004a, AN, 325, 171

Sánchez, S. F., Christensen, L., Becker, T., et al. 2004b, AN, 325, 112

Sánchez, S. F., \& González-Serrano, J. I. 2003, A\&A, 406, 435

Sánchez, S. F., Jahnke, K., Wisotzki, L., et al. 2004, ApJ, 614, 586

Sanders, D. B., \& Mirabel, I. F. 1996, ARA\&A, 34, 749

Sanders, D. B., Soifer, B. T., Elias, J. H., Neugebauer, G., \& Matthews, K. 1988, ApJ, 328, L35

Sekiguchi, K., \& Wolstencroft, R. D. 1992, MNRAS, 255, 581

Toomre, A., \& Toomre, J. 1972, ApJ, 178, 623

van den Broek, A. C., van Driel, W., de Jong, T., et al. 1991, A\&AS, 91,61

Veilleux, S., \& Osterbrock, D. E. 1987, ApJS, 63, 295 